\title{
Boosted Dark Matter Interpretation of the XENON1T Excess
}

\author{
Bartosz Fornalø ${ }^{1}$ Pearl Sandick $\odot,{ }^{1}$ Jing Shu $\odot,{ }^{2,3,4,5,6,7, *}$ Meng $S u,{ }^{8,9}$ and Yue Zhao ${ }^{1, \dagger}$ \\ ${ }^{1}$ Department of Physics and Astronomy, University of Utah, Salt Lake City, Utah 84112, USA \\ ${ }^{2}$ CAS Key Laboratory of Theoretical Physics, Institute of Theoretical Physics, Chinese Academy of Sciences, \\ Beijing 100190, China \\ ${ }^{3}$ School of Physical Sciences, University of Chinese Academy of Sciences, Beijing 100049, China \\ ${ }^{4}$ CAS Center for Excellence in Particle Physics, Beijing 100049, China \\ ${ }^{5}$ Center for High Energy Physics, Peking University, Beijing 100871, China \\ ${ }^{6}$ School of Fundamental Physics and Mathematical Sciences, Hangzhou Institute for Advanced Study, \\ University of Chinese Academy of Sciences, Hangzhou 310024, China \\ ${ }^{7}$ International Centre for Theoretical Physics Asia-Pacific, Beijing/Hangzhou, China \\ ${ }^{8}$ Department of Physics, The University of Hong Kong, Hong Kong SAR 999077, China \\ ${ }^{9}$ Laboratory for Space Research, The University of Hong Kong, Hong Kong SAR 999077, China
}

(Received 26 June 2020; accepted 9 September 2020; published 12 October 2020)

\begin{abstract}
We propose boosted dark matter (BDM) as a possible explanation for the excess of keV electron recoil events observed by XENON1T. BDM particles have velocities much larger than those typical of virialized dark matter, and, as such, BDM-electron scattering can naturally produce keV electron recoils. We show that the required BDM-electron scattering cross sections can be easily realized in a simple model with a heavy vector mediator. Though these cross sections are too large for BDM to escape from the Sun, the BDM flux can originate from the Galactic Center or from halo dark matter annihilations. Furthermore, a daily modulation of the BDM signal will be present, which could not only be used to differentiate it from various backgrounds but would also provide important directional information for the BDM flux.
\end{abstract}

DOI: 10.1103/PhysRevLett.125.161804

Introduction.-The XENON1T experiment has recently reported an excess in their low energy electron recoil data, appearing between $2-3 \mathrm{keV}$ [1]. Three possible explanations are proposed in [1] for this excess. The first possible explanation is beta decays of tritium, which currently can neither be confirmed nor ruled out due to our lack of knowledge of the tritium concentration. The other two possible explanations pursued in [1] are solar axions and anomalous neutrino interactions. However, the preferred values of couplings in the latter two cases have already been ruled out by existing astrophysical constraints, particularly from stellar cooling [2-5]. Furthermore, it is well known that the observed excess in electron recoils cannot be due to typical dark matter (DM) particles scattering on electrons, since a DM particle moving at virial velocity, i.e., $\mathcal{O}\left(10^{-3}\right) c$, will result in an energy deposition that is much smaller than the $\mathrm{keV}$-scale excess. Therefore it is interesting and important to explore other possible explanations for the observed excess.

Published by the American Physical Society under the terms of the Creative Commons Attribution 4.0 International license. Further distribution of this work must maintain attribution to the author(s) and the published article's title, journal citation, and DOI. Funded by SCOAP.
Many theoretical models predict the existence of boosted dark matter (BDM) in our Universe. With BDM typical velocities much larger than the virial velocity, BDM models are therefore generically capable of producing an excess of electron recoils such as the one observed. For example, the DM-induced nucleon decay process studied in [6] produces BDM in its final state, while semiannihilation [7] and multicomponent [8] DM models also yield BDM fluxes from the Sun or the Galactic Center (GC). BDM flux searches have been proposed for large volume neutrino experiments, and interesting parameter space has been covered by existing experiments such as Super Kamiokande [9], ProtoDUNE [10], and IceCube [11,12] or will be covered by future experiments such as DUNE [13-15].

In this Letter, we propose that the $\mathrm{keV}$ excess in electron recoils observed by XENON1T could be due to BDM scattering on electrons. If $m_{\mathrm{BDM}} \gg m_{e}$, a typical energy deposition from BDM-electron scattering of $\sim$ few $\mathrm{keV}$ implies the velocity of the BDM must be $\mathcal{O}(0.1) c$, thus only mildly boosted. Here we present two example models and consider the flux of BDM particles from the Milky Way halo and the Sun. We find that including BDM-electron recoils can significantly improve the fit to the data relative to the background only hypothesis and that the required scattering cross section can be naturally explained by 
models with a vector mediator. We highlight that our BDM signals enjoy a daily modulation. Such a unique feature can be used to distinguish the signal proposed here from various backgrounds, as well as many other new physics interpretations of this excess [16-18]. Furthermore, the phase and the amplitude of the modulation can be used to extract crucial information about the BDM, e.g., its flux direction, providing essential guidance for a future experimental analysis. Finally, we note that this work is an important addition to the existing BDM literature, extending the scientific goals of a DM direct detection experiment to search for BDM in the mildly boosted regime.

Models for boosted dark matter.-DM particles moving at virial velocity are not capable of depositing energy as large as a few $\mathrm{keV}$ when scattering on electrons. However, in the BDM scenario, some fraction of DM particles are boosted such that their velocities are much larger than those typical of virialized DM. Indeed, BDM scattering on electrons would therefore result in higher energy recoil signals. The existence of a BDM flux is a generic prediction in many well-motivated DM models.

The first example model is the semiannihilation DM model [7]. In this case, $\mathrm{DM} \chi$ carries a $Z_{3}$ symmetry, and the BDM flux is produced through

$$
\chi+\chi \rightarrow \bar{\chi}+X .
$$

Here $X$ can be a standard model particle or can eventually decay to standard model particles. The boost factor of the $\bar{\chi}$ in the final state is $\gamma_{\chi}=\left(5 m_{\chi}^{2}-m_{X}^{2}\right) / 4 m_{\chi}^{2}$. In the limit of $m_{\chi} \gg m_{X}$, the boost factor reaches its maximum value of 1.25 .

The second BDM model we study here is the twocomponent DM model (see, e.g., [19] and subsequent work). In this case, two particles, $\psi_{A}$ and $\psi_{B}$, are both stable. We assume $\psi_{A}$ is heavier and is the dominant component of the DM. The annihilation of $\psi_{A}$ particles produces boosted $\psi_{B}$ particles in the final state

$$
\psi_{A}+\bar{\psi}_{A} \rightarrow \psi_{B}+\bar{\psi}_{B} .
$$

The boost factor of the $\psi_{B}$ particles is simply the mass ratio, i.e., $\gamma_{B}=m_{A} / m_{B}$.

Boosted dark matter sources.-There are two promising sources to generate the BDM flux: annihilation in the GC or halo and capture and annihilation in the Sun. Here we summarize the expected flux from each source.

Assuming the DM follows a Navarro-Frenk-White profile [20], the BDM flux from the full sky can be written as $[8]$

$$
\begin{aligned}
\Phi_{\mathrm{gal}}^{\mathrm{BDM}}= & 1.6 \times 10^{-6} \mathrm{~cm}^{-2} \mathrm{~s}^{-1} \\
& \times\left(\frac{\left\langle\sigma_{\mathrm{ann}} v\right\rangle}{5 \times 10^{-26} \mathrm{~cm}^{3} \mathrm{~s}^{-1}}\right)\left(\frac{10 \mathrm{GeV}}{m_{\mathrm{DM}}}\right)^{2} .
\end{aligned}
$$

Here $\left\langle\sigma_{\mathrm{ann}} v\right\rangle$ is the total thermally averaged DM annihilation cross section, which, under the assumption of DM thermal history, provides the correct relic abundance. We note that $\mathrm{DM}$ can also be produced in a nonthermal manner, in which case the DM annihilation cross section can be larger, leading to a larger BDM flux from the GC. Though the DM density peaks toward the GC, since XENON1T cannot distinguish the direction of the incoming DM particle, all sky directions should be included.

A second source for the BDM flux is the Sun. If DM particles scatter on nuclei and are captured by the Sun, DM can accumulate in the Sun's core over time. The solar capture rate can be approximated by [21]

$$
\begin{aligned}
C\left(m_{\mathrm{DM}}, \sigma_{\text {nucl }}\right) \simeq & 2 \times 10^{22} \mathrm{~s}^{-1} \\
& \times\left(\frac{\sigma_{\text {nucl }}}{10^{-42} \mathrm{~cm}^{2}}\right)\left(\frac{10 \mathrm{GeV}}{m_{\mathrm{DM}}}\right)^{2} .
\end{aligned}
$$

The cross section $\sigma_{\text {nucl }}=10^{-42} \mathrm{~cm}^{2}$ is comparable to the upper limit on the DM-neutron scattering imposed by spin-dependent DM direct detection experiments [22]. For simplicity, this approximation assumes that the DMnucleon scattering cross section does not depend on the relative velocity at leading order. If the leading order cross section has a $v^{2}$ dependence, the DM capture rate can be enhanced by a factor of $\sim 25$. We also note that our choice of benchmark value for the DM-nucleon scattering cross section of $\sigma_{\text {nucl }}=10^{-42} \mathrm{~cm}^{2}$ corresponds to the bound on the spin-dependent scattering cross section obtained by DM direct detection experiments (see, e.g., [23]).

For typical choices of DM scattering and annihilation cross sections, the Sun will reach a capture-annihilation equilibrium [24]. In this case, the DM annihilation cross section becomes irrelevant, and the BDM flux is fully determined by the DM capture rate, which is characterized by the DM-nucleon scattering cross section, $\sigma_{\text {nucl }}$. Thus, the BDM flux can be written as

$$
\begin{aligned}
\Phi_{\mathrm{Sun}}^{\mathrm{BDM}} & =\frac{C\left(m_{\mathrm{DM}}, \sigma_{\text {nucl }}\right)}{4 \pi(\mathrm{A} . \mathrm{U} .)^{2}} \\
& =7.2 \times 10^{-6} \mathrm{~cm}^{-2} \mathrm{~s}^{-1}\left(\frac{\sigma_{\text {nucl }}}{10^{-42} \mathrm{~cm}^{2}}\right)\left(\frac{10 \mathrm{GeV}}{m_{\mathrm{DM}}}\right)^{2},
\end{aligned}
$$

where A.U. is an astronomical unit. We note that there is an important subtlety regarding the BDM flux from the Sun, to which we will return shortly.

Signal rate.-For a given BDM flux, one can estimate the total number of signal events as

$$
\begin{aligned}
N_{\text {sig }} & =Z^{\prime} n_{\mathrm{Xe}} V T \sigma_{\text {elec }} \Phi^{\mathrm{BDM}} \\
& =Z^{\prime} \frac{M_{\mathrm{det}} T}{m_{\mathrm{Xe}}} \times \sigma_{\text {elec }} \times \Phi^{\mathrm{BDM}} .
\end{aligned}
$$


Here $n_{\mathrm{Xe}}$ is the number density of xenon atoms in the detector, $M_{\text {det }}$ and $V_{\text {det }}$ are the fiducial mass and volume of the detector, $T$ is the total operation time, and $\sigma_{\text {elec }}$ is the BDM-electron scattering cross section. The exposure $M_{\text {det }} T$ for the XENON1T data presented in [1] is 0.65 tonne-years. $Z^{\prime}$ is the effective number of electrons in xenon that undergo recoils. In particular, electrons on shells $K$ and $L$ have binding energies $E_{b}>4.5 \mathrm{keV}$ and cannot be knocked out by DM with kinetic energies relevant for the excess. For shell $M$, the binding energies fall within the range $0.68-1.15 \mathrm{keV}$, leading to contributions at the lower part of the spectrum. Finally, the electrons on shells $N$ and $O$ have $E_{b}<0.22 \mathrm{keV}$ and contribute almost like free particles. This leads to $Z^{\prime} \sim 40$.

In order to explain the excess observed by XENON1T, the number of the signal events needs to be $\mathcal{O}(100)$. This translates to a BDM-electron scattering cross section of

$$
\sigma_{\text {elec }}=3 \times 10^{-29} \mathrm{~cm}^{2}\left(\frac{10^{-6} \mathrm{~cm}^{-2} \mathrm{~s}^{-1}}{\Phi^{\mathrm{BDM}}}\right)\left(\frac{N_{\mathrm{sig}}}{100}\right) .
$$

This provides a rough prediction for the BDM-electron scattering cross section.

Now, let us examine whether it is reasonable to expect $\sigma_{\text {elec }}$ to be as large as $\mathcal{O}\left(10^{-29}-10^{-28}\right) \mathrm{cm}^{2}$. If a BDM particle scatters with an electron through a vector mediator whose mass is much larger than the typical momentum transfer, the scattering cross section can be written as [25]

$$
\sigma_{\text {elec }}=\frac{g_{\mathrm{BDM}}^{2} g_{e}^{2} m_{e}^{2}}{\pi m_{\mathrm{med}}^{4}},
$$

where $g_{\mathrm{BDM}}\left(g_{e}\right)$ is the coupling between the mediator and $\mathrm{BDM}$ (electron). As a benchmark, consider $g_{\mathrm{BDM}}=1.1$, $g_{e}=10^{-5}, \quad m_{\mathrm{BDM}}=10 \mathrm{GeV}$, and $m_{\mathrm{med}}=0.1 \mathrm{MeV}$, which results in $\sigma_{\text {elec }}=4 \times 10^{-29} \mathrm{~cm}^{2}$. Thus, for reasonable parameter values, a cross section as large as $\sigma_{\text {elec }}=$ $\mathcal{O}\left(10^{-29}-10^{-28}\right) \mathrm{cm}^{2}$ can be obtained [26].

Existing astrophysical observations and terrestrial experiments are a potential source of strong constraints. However, all of them are avoided in our benchmark model. The mediator mass $m_{\text {med }}=0.1 \mathrm{MeV}$ is at the heavier end of the stellar cooling reach and, with a coupling to electrons $g_{e}=10^{-5}$, these particles cannot escape from the core [30], circumventing any related constraints. Supernova bounds assume that the dominant production is through bremsstrahlung in nucleon scattering processes [31], which are not present in the benchmark scenario. Constraints from beam dump dark photon searches do not extend to masses $m_{\text {med }}<1 \mathrm{MeV}$ [32]. Finally, the early production of mediators introduces deviations on $N_{\text {eff }}$. However a late phase transition in the dark sector [33-35] may modify the thermal history and relax this constraint.

One important question for mildly boosted DM with a large $\sigma_{\text {elec }}$ is whether the BDM can penetrate the Sun after its production near the core. For a solar core density of $150 \mathrm{~g} / \mathrm{cm}^{3}$, the free streaming length in the Sun is

$$
L_{\mathrm{fs}, S} \simeq 1 \mathrm{~m} \times\left(\frac{10^{-28} \mathrm{~cm}^{2}}{\sigma_{\text {elec }}}\right),
$$

and for each scattering the momentum transfer is $\mathcal{O}(10-100) \mathrm{keV}$. With a solar core radius of $1.4 \times 10^{5} \mathrm{~km}$, it is unlikely that the BDM produced near the center of the Sun will escape. Thus, if the XENON1T excess is explained by BDM, the flux is likely to be produced in the $\mathrm{GC}$, as there would be no appreciable flux from the Sun for the required BDM-electron scattering cross section. We note that simple variations of BDM models considered here can avoid the difficulty of escaping the Sun, which we will comment on later.

Similarly, let us also calculate how far the BDM can propagate in the Earth. Taking the average Earth density as $5.5 \mathrm{~g} / \mathrm{cm}^{3}$, the free streaming length in the Earth is

$$
L_{\mathrm{fs}, E} \simeq 60 \mathrm{~m} \times\left(\frac{10^{-28} \mathrm{~cm}^{2}}{\sigma_{\text {elec }}}\right) .
$$

The XENON1T experiment operates underground at a depth of about $1600 \mathrm{~m}$. While the average rock density above XENON1T is somewhat smaller than the average Earth density quoted here, the average used here is sufficient for the following order-of-magnitude estimates. As discussed above, BDM has a velocity of $\mathcal{O}(0.1) c$. If every BDM-electron collision reduces the BDM momentum by $\mathcal{O}(10-100) \mathrm{keV}$, a BDM particle whose mass is smaller than $\mathcal{O}(0.01-0.1) \mathrm{GeV}$ may not be able to reach the detector, assuming the benchmark BDM-electron scattering cross section at $10^{-28} \mathrm{~cm}^{2}$. Furthermore, if BDM propagates for a distance comparable to the Earth's radius of $6000 \mathrm{~km}$, its momentum may be reduced by $\mathcal{O}(1-10) \mathrm{GeV}$. If the BDM mass is below $\mathcal{O}(10-100) \mathrm{GeV}$, there is a high chance that it cannot fully penetrate the Earth. This leads to a daily modulation of the DM signal if the BDM flux is dominantly from the GC [36]. This feature may provide an important handle to reduce various backgrounds and can potentially be used as a smoking gun signature for BDM discovery. More details about daily modulation will be provided in a later discussion.

Energy deposition distribution.-Finally, we study the electron recoil energy distribution at XENON1T resulting from BDM-electron scattering in the benchmark scenario discussed above. The energy deposition required to fit the excess is $\sim$ few $\mathrm{keV}$. In the limit $m_{\mathrm{BDM}} \gg m_{e}$, which is applicable in our case, the electron recoil energy is $E_{e} \leq 2 m_{e} v_{\mathrm{BDM}}^{2}$. The corresponding differential cross section is approximately a flat function, which can be written as

$$
\frac{d \sigma_{\text {elec }}}{d E_{e}}=\text { const } \times \Theta\left(2 m_{e} v_{\mathrm{BDM}}^{2}-E_{e}\right),
$$

where $\Theta$ is the Heaviside step function. 


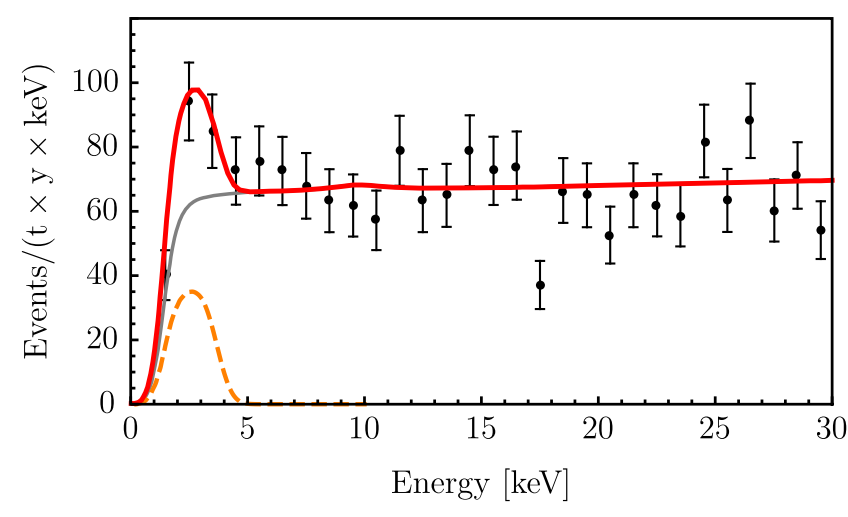

FIG. 1. The energy spectrum of electrons for a benchmark with $m_{\mathrm{BDM}}=10 \mathrm{GeV}, v_{\mathrm{BDM}}=0.06 c, \sigma_{\text {elec }}=4 \times 10^{-29} \mathrm{~cm}^{2}$, and BDM flux $\Phi^{\mathrm{BDM}}=10^{-6} \mathrm{~cm}^{-2} \mathrm{~s}^{-1}$. The dashed orange line represents the contribution from BDM-electron scattering after including the energy resolution and detection efficiency. The red line shows the total electron energy distribution at XENON1T.

To derive the expected signal at XENON1T, we convolute this differential cross section with a Gaussian of width $\Delta=0.5 \mathrm{keV}$, corresponding to the detector energy resolution in the $\sim \mathrm{keV}$ region [38], and weight the cross section by the detector efficiency [1].

In Fig. 1, we demonstrate how BDM-electron scattering can improve the fit to data. Here we choose as a benchmark $m_{\mathrm{BDM}}=10 \mathrm{GeV}, v_{\mathrm{BDM}}=0.06 c$, and $\sigma_{\text {elec }}=4 \times 10^{-29} \mathrm{~cm}^{2}$. The BDM-electron scattering produces a bump, denoted by the orange dashed line, in the electron recoiling energy spectrum. The total electron spectrum at XENON1T is given by the red line. For the benchmark model under consideration, adding contributions from BDM-electron scattering can provide an excellent fit to data.

Daily modulation.-DM signals in direct detection experiments may present nontrivial time dependence as first studied in [39], where it was proposed that an annual modulation in scattering events could arise due to the Earth's motion around the Sun. A daily modulation in DM-nucleus scattering due to the Earth's shielding of a terrestrial detector from the DM wind was explored in [40], and similar cases have been further studied in, e.g., [41-44].

Given the sizable DM-electron scattering cross section necessary to explain the XENON1T excess, a daily modulation of the signal, due to the anisotropy of the BDM flux and the dynamics of Earth's shielding, is a natural prediction. Indeed, a similar daily modulation due to Earth's shielding of a flux of cosmic ray boosted DM from the GC was recently studied in [45].

In this section, we demonstrate some features of the expected daily modulation that would allow extraction of information about the BDM model from the XENON1T signal. We note that the estimates presented here are crude but serve to illustrate the capability of learning about BDM from a direct detection signal. Specifically, we consider the
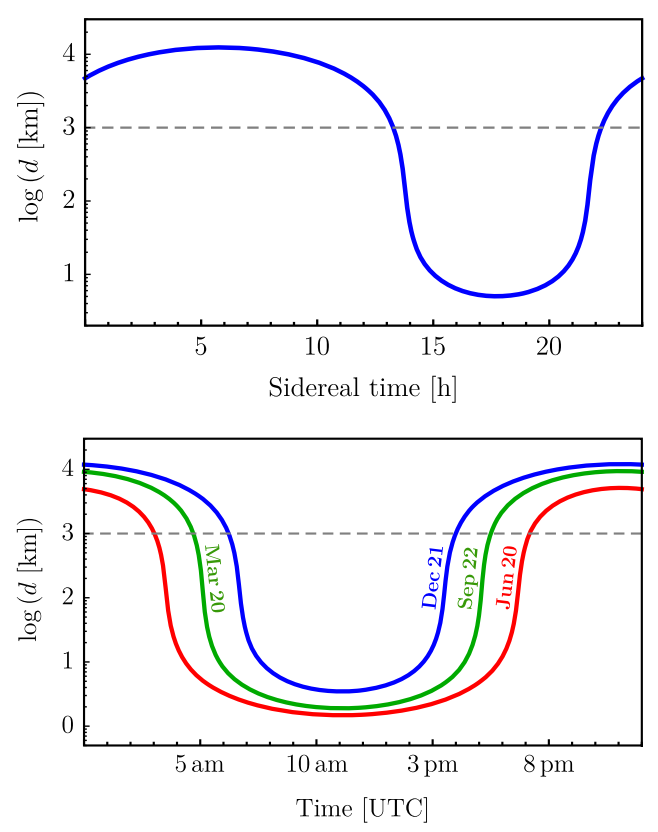

FIG. 2. Upper: The depth that a BDM particle from the GC must penetrate to reach the XENON1T detector as a function of the sidereal time. Lower: The depth that a BDM particle from the Sun must penetrate to reach the XENON1T detector as a function of the universal time for four dates throughout the year. The gray dashed line in each panel indicates a depth of $1000 \mathrm{~km}$, as in Eq. (12).

Earth to be a sphere of constant density and assume that the XENON1T detector is buried $1.6 \mathrm{~km}$ beneath the surface at the location of the Gran Sasso National Lab. A more extensive analysis is beyond the scope of this study but will ultimately be critical for investigating a BDM origin of the XENON1T excess.

First, we consider a BDM flux from the GC. The XENON1T lab rotates around the Earth's axis once each sidereal day, and its position can be parameterized by the sidereal time (ST). In the upper panel of Fig. 2, we show the depth of the Earth $d$ that a BDM particle must penetrate in order to reach the XENON1T detector as a function of ST. It is straightforward to convert ST to the local time in Gran Sasso at any given time of the year; the position of the minimum depth shifts by $\sim$ two hours per month in terms of the local time. The depth $d$ reaches its minimum value when the detector is oriented with the least Earth shielding in the direction of the source, which in this case is the GC. Therefore, the directional information is encoded in the position of the signal peak, i.e., the phase. Even for a detector such as XENON1T, with no design capability to distinguish directionality, it may be possible to identify the source of a BDM flux.

It may happen, however, that the dominant component of the BDM flux does not come from the GC but rather from another direction in the sky. As discussed above, BDM particles arising in the simplest scenarios are unlikely to 
escape the Sun due to their interactions with electrons, but it is not difficult to construct a BDM model for which a substantial flux from the Sun is expected. For example, in a multicomponent DM model, consider the possibility that $\psi_{A}$ particles are captured by the Sun and annihilate each other through the decay chain:

$$
\psi_{A}+\bar{\psi}_{A} \rightarrow \phi+\phi^{*} \rightarrow 2 \psi_{B}+2 \bar{\psi}_{B},
$$

where $\phi$ is an intermediate particle whose decay length is comparable to the solar radius. The $\psi_{B}$ particles arising from $\phi$ decay are therefore produced outside the Sun. In this case, the BDM particles would point back to the Sun and could still have a large scattering cross section with electrons.

In the lower panel of Fig. 2, we show the depth of the Earth $d$ that the BDM must penetrate as a function of the Universal Coordinated Time (UTC), assuming the Sun is the source of the BDM flux. While the position of the signal peak remains constant, the shape of the modulation varies throughout the year. In the winter, when the days are shortest in the northern hemisphere, XENON1T experiences its shortest daily exposures with minimal shielding, as shown by the relatively short daytime dip in the blue curve in Fig. 2. In the summer, on the other hand, the daily exposure with low shielding is the longest, as demonstrated by the red curve in Fig. 2. We note that these general conclusions hold independently of the details of the XENON1T position or the Earth density modeling.

Finally, it is instructive to estimate the depth at which most BDM particles are stopped. We assume that each BDM-electron scattering reduces the BDM kinetic energy by $\sim 3 \mathrm{keV}$. This implies that the BDM signal approaches zero when

$d_{e} \gtrsim 1000 \mathrm{~km}\left(\frac{10^{-28} \mathrm{~cm}^{2}}{\sigma_{\text {elec }}}\right)\left(\frac{m_{\mathrm{BDM}}}{10 \mathrm{GeV}}\right)\left(\frac{v_{\mathrm{BDM}}}{0.1 c}\right)^{2}$.

As an illustration, consider the survival probability $P$ as a function of the penetration depth $d$ to be modeled by a Heaviside step function $P(d)=\Theta\left(d_{e}-d\right)$. The daily modulation of the signal is determined by the survival probability of the incoming BDM particles. To make a realistic prediction for the signal, care must be taken when modeling the position and overburden of the detector. Furthermore, for the BDM flux from the Sun, one must also account for the seasonal variation of $d(t)$, as described above. A detailed analysis, including a realistic simulation of the survival probability function for BDM-electron interactions is left for future study.

Again, we emphasize that even for a DM direct detection experiment such as XENON1T, which is not designed to detect the directionality of scattering events [46], the phase of the daily modulation could be used to extract directional information for the BDM flux. The GC and the Sun may be the most likely sources for a BDM flux, but it is also conceivable that a BDM flux comes from a nearby concentration of DM, such as a DM subhalo or a minicluster. Indeed, the phase of the daily modulation could in principle take any value and could be used to identify the flux direction or source.

Conclusion.-In this Letter, we explain the XENON1T excess reported in [1] as a signal of BDM-electron scattering. Such an interaction can be naturally introduced through a vector mediator whose mass is much larger than the typical momentum transfer in the scattering. With reasonable choices of parameters, cross sections of the magnitude necessary to explain the excess can be easily obtained and significantly improve the fit to the XENON1T data. We consider two possible sources for the BDM flux: the GC or halo and the Sun. We find that the required BDM-electron scattering cross section is large enough that the BDM particles studied here would not escape from the Sun. Thus, if XENON1T is indeed observing BDM, either it must come from the GC or the Milky Way halo or the BDM model must be more complicated than the minimal scenario we highlight here.

The predicted daily modulation of the signal opens a brand new strategy to investigate possible sources for the excess. The daily modulation will not only improve the signal significance and help discriminate against backgrounds such as beta decays of tritium, but it also provides a unique opportunity to determine the origin of the BDM flux. The maximum of the BDM flux occurs when the detector is oriented with the least Earth shielding in the direction of the source. Therefore, the phase of this daily modulation could potentially resolve the direction of the BDM flux, presenting a novel opportunity to discover the true nature of dark matter.

Finally, we note that the BDM-electron scattering interpretation of the XENON1T excess can be investigated also in other experiments. This might be difficult at PandaX, since tritium is injected into the detector for calibration purposes, and the only way to overcome the resulting background is by studying the daily modulation of the signal. However, future experiments such as XENONnT or PandaX-4T, without tritium doping, are expected to have a better sensitivity, provided they maintain similar energy thresholds for the electron recoil channel.

We thank Jianglai Liu and Qiang Yuan for helpful discussions. The work of P.S. is supported by NSF Grant No. PHY-1720282. B.F. and Y.Z. are supported by the U.S. Department of Energy under Award No. DE-SC0009959. J.S. is supported by the National Natural Science Foundation of China (NSFC) under Grants No. 11947302, No. 11690022, No. 11851302, No. 11675243, and No. 11761141011 and also supported by the Strategic Priority Research Program of the Chinese Academy of Sciences under Grants No. XDB21010200 and No. XDB23000000. M. S. is supported by a grant from 
the General Research Fund by the Research Grants Council of Hong Kong SAR (Ref. 17305517).

Note added-We note that there are four other papers $[17,47-49]$ to appear on the same issue aiming to explain the XENON1T excess. Particularly [47] exhibits similar daily modulation feature as suggested in this study.

* Corresponding author.

jshu@itp.ac.cn

Corresponding author. zhaoyue@physics.utah.edu

[1] E. Aprile et al. (XENON Collaboration), arXiv:2006.09721.

[2] G. G. Raffelt, Stars as Laboratories for Fundamental Physics (Chicago University Press, Chicago, 1996).

[3] M. Giannotti, I. G. Irastorza, J. Redondo, A. Ringwald, and K. Saikawa, J. Cosmol. Astropart. Phys. 10 (2017) 010.

[4] A. H. Corsico, L. G. Althaus, M. M. M. Bertolami, S. Kepler, and E. Garcia-Berro, J. Cosmol. Astropart. Phys. 08 (2014) 054.

[5] S. A. Díaz, K.-P. Schröder, K. Zuber et al., arXiv:1910.10568.

[6] J. Huang and Y. Zhao, J. High Energy Phys. 02 (2014) 077.

[7] F. D'Eramo and J. Thaler, J. High Energy Phys. 06 (2010) 109.

[8] K. Agashe, Y. Cui, L. Necib, and J. Thaler, J. Cosmol. Astropart. Phys. 10 (2014) 062.

[9] C. Kachulis et al. (Super-Kamiokande Collaboration), Phys. Rev. Lett. 120, 221301 (2018).

[10] A. Chatterjee, A. De Roeck, D. Kim, Z. G. Moghaddam, J. C. Park, S. Shin, L. H. Whitehead, and J. Yu, Phys. Rev. D 98, 075027 (2018).

[11] A. Bhattacharya, R. Gandhi, A. Gupta, and S. Mukhopadhyay, J. Cosmol. Astropart. Phys. 05 (2017) 002.

[12] J. Kopp, J. Liu, and X. P. Wang, J. High Energy Phys. 04 (2015) 105.

[13] J. Berger, Y. Cui, M. Graham, L. Necib, G. Petrillo, D. Stocks, Y. T. Tsai, and Y. Zhao, arXiv:1912.05558.

[14] C. Argelles et al., arXiv:1907.08311.

[15] B. Abi et al. (DUNE Collaboration), arXiv:2002.03005.

[16] J. Smirnov and J. F. Beacom, Phys. Rev. Lett. 125, 131301 (2020).

[17] F. Takahashi, M. Yamada, and W. Yin, this issue, Phys. Rev. Lett. 125, 161801 (2020).

[18] K. Kannike, M. Raidal, H. Veerme, A. Strumia, and D. Teresi, arXiv:2006.10735.

[19] C. Boehm, P. Fayet, and J. Silk, Phys. Rev. D 69, 101302 (2004).

[20] J. F. Navarro, C. S. Frenk, and S. D. White, Astrophys. J. 462, 563 (1996).

[21] J. Berger, Y. Cui, and Y. Zhao, J. Cosmol. Astropart. Phys. 02 (2015) 005.

[22] E. Aprile et al. (XENON Collaboration), Phys. Rev. Lett. 122, 141301 (2019).
[23] J. Aalbers et al. (DARWIN Collaboration), J. Cosmol. Astropart. Phys. 11 (2016) 017.

[24] For a detailed discussion, please see [21].

[25] A. Joglekar, N. Raj, P. Tanedo, and H. B. Yu, Phys. Lett. B809, 135767 (2020).

[26] In semiannihilation models, there is only one species of DM particle, and the DM capture may be dominantly caused by DM-electron scattering. This will modify the capture rate estimation in Eq. (5). In addition, when the BDM particle is the same as the DM particle, such a strong interaction with electrons has already been ruled out by DM direct detection experiments, e.g., [27-29], unless one introduces nontrivial velocity dependence in the DM-electron cross section. Thus, semiannihilation models are disfavored in explaining the XENON1T excess.

[27] R. Essig, A. Manalaysay, J. Mardon, P. Sorensen, and T. Volansky, Phys. Rev. Lett. 109, 021301 (2012).

[28] E. Aprile et al. (XENON Collaboration), Phys. Rev. Lett. 123, 251801 (2019).

[29] L. Barak et al. (SENSEI Collaboration), arXiv:2004.11378.

[30] J. Redondo, J. Cosmol. Astropart. Phys. 07 (2008) 008.

[31] J. H. Chang, R. Essig, and S. D. McDermott, J. High Energy Phys. 01 (2017) 107.

[32] R. Essig et al., arXiv:1311.0029.

[33] Z. Chacko, L. J. Hall, S. J. Oliver, and M. Perelstein, Phys. Rev. Lett. 94, 111801 (2005).

[34] H. Davoudiasl and C. W. Murphy, Phys. Rev. Lett. 118, 141801 (2017).

[35] Y. Zhao, Phys. Rev. D 95, 115002 (2017).

[36] Such Earth shielding effect is under investigation in [37].

[37] H. Day, D. Liu, M. A. Luty, and Y. Zhao (to be published).

[38] E. Aprile et al. (XENON Collaboration), Eur. Phys. J. C 80, 785 (2020).

[39] A. Drukier, K. Freese, and D. Spergel, Phys. Rev. D 33, 3495 (1986).

[40] J. Collar and F. Avignone, Phys. Lett. B 275, 181 (1992).

[41] F. Hasenbalg, D. Abriola, F. Avignone, J. Collar, D. Di Gregorio, A. Gattone, H. Huck, D. Tomasi, and I. Urteaga, Phys. Rev. D 55, 7350 (1997).

[42] C. Kouvaris and I. M. Shoemaker, Phys. Rev. D 90, 095011 (2014).

[43] B. J. Kavanagh, R. Catena, and C. Kouvaris, J. Cosmol. Astropart. Phys. 01 (2017) 012.

[44] T. Emken, R. Essig, C. Kouvaris, and M. Sholapurkar, J. Cosmol. Astropart. Phys. 09 (2019) 070.

[45] S. F. Ge, J. L. Liu, Q. Yuan, and N. Zhou, arXiv:2005.09480.

[46] F. Mayet et al., Phys. Rep. 627, 1 (2016).

[47] N. F. Bell, J. B. Dent, B. Dutta, S. Ghosh, J. Kumar, and J. L. Newstead, preceding Letter, Phys. Rev. Lett. 125, 161803 (2020).

[48] A. Bally, S. Jana, and A. Trautner, this issue, Phys. Rev. Lett. 125, 161802 (2020).

[49] J. Bramante and N. Song, following Letter, Phys. Rev. Lett. 125, 161805 (2020) 